\title{
Análisis estadístico de la producción de un biocontrolador de la mosca blanca en plantas de frijol bajo invernadero
}

\author{
Statistical Analysis of the Production of a Biocontroller of Whiteflies \\ in Greenhouse Bean Plants
}

Marco Antonio Día目

U7500071@unimilitar.edu.co
Fernando Cantor

fernando.cantor@unimilitar.edu.co

Daniel Rodríguezc

U7500061@unimilitar.edu.co

\section{Resumen}

La mosca blanca de los invernaderos Trialeurodes vaporariorum es una de las plagas agrícolas de mayor importancia en la sabana de Bogotá. Para su manejo se han implementado diferentes estrategias de control, como el uso de enemigos naturales, siendo esta una alternativa ecológica. La avispa parasitoidea Encarsia formosa se ha reconocido como controlador natural de esta plaga y su uso ha sido documentado mundialmente como uno de los casos exitosos de control biológico más reconocidos. En los últimos años la Universidad Militar Nueva Granada ha establecido una cría semi-comercial de este controlador con fines de distribución y venta agrícola en la sabana de Bogotá. Con el fin de optimizar el proceso de producción de la cría, se realizó un análisis estadístico recopilando los datos de producción de los meses de agosto a diciembre del 2010. Se estableció el nivel máximo, mínimo y el promedio de producción a partir de la información recolectada. Posteriormente, se realizó una fase experimental para encontrar la relación entre el área foliar de una planta de frijol atacada y el porcentaje de infestación de la plaga, la preferencia de oviposición de E. formosa en la hoja de frijol infestada con T. vaporariorum y la relación entre el número de avispas usadas en la cría con el número de avispas producidas. Se encontró que no existe una relación entre el área foliar con el número de individuos de la plaga pero sí una preferencia de alimentación hacia la zona central de la hoja. Finalmente se ajustó una regresión

\footnotetext{
${ }^{a}$ Estudiante. Maestría en Biología Aplicada. Laboratorio de Control Biológico. Facultad de Ciencias. Universidad Militar Nueva Granada.

b Docente. Programa Biología Aplicada, Laboratorio de Control Biológico, Facultad de Ciencias, Universidad Militar Nueva Granada.

${ }^{\mathrm{c}}$ Docente. Programa Biología Aplicada, Laboratorio de Control Biológico, Facultad de Ciencias, Universidad Militar Nueva Granada.
} 
polinomial entre la eficiencia de parasitación de la avispa con la población de la misma. Se concluye que para mejorar la producción es necesario liberar una cantidad menor de avispas de las establecidas originalmente.

Palabras clave: encarsia formosa, trialeurodes vaporariorum, cría masiva, regresión polinomial, ANOVA, control biológico.

\begin{abstract}
The greenhouse whitefly Trialeurodes vaporariorum, is one of the majorcroppestsin Colombia. Several strategies have been used in order to control this pest,being one of them the use of natural enemies, which is an environmentally friendly strategy. The parasitic wasp Encarsia formosaacts as a natural enemy of whiteflies and it have been documented as one of the most successful cases of biological control around the world.In the last years, the Nueva Granada Military University has established a half-commercial production unit of this wasp with purpose of commercial and agricultural distribution in the savannah of Bogotá. In order to optimize the mass rearing process, a statistical analysis was performed using the production data between August and December of 2010. Using this information, the maximum, minimum and mean level of production was established. Later, an experimental faze was performed in order to find the relation of the leaf area of a bean plant attacked and the proportion of pest infestation, the oviposition preference of $E$. formosa in a bean leaf infested with $T$. vaporariorum and the relation of the number of wasp used in the mass rearing process and the number of wasp produced. The data were processed using the statistical package SAS INSTITUTE INC (2008). There isn't a relation between the leaf area and the number of whiteflies in a leaf but there is a feeding preference towards the center of the leaf. Finally, regression analysis was made between the waspparasitization efficiency and the population density of the wasp. In order to improve the production is necessary release a lower number of wasps rather than the originally established.
\end{abstract}

Key words: Encarsia formosa, Trialeurodes vaporariorum, mass-rearing, polynomial regression, ANOVA, biological control.

\title{
1. Introducción
}

Las plagas agrícolas siempre han sido una constante en cualquier cultivo, ya sea en campo o en invernadero. Esto se debe al desequilibrio causado, por implementar un sistema de diversidad reducida, en un ambiente natural. En nuestro país, el manejo de estas plagas se realiza normalmente con productos químicos, que a pesar de su eficacia, traen consecuencias graves a corto y largo plazo sobre el medio ambiente.

En un país como Colombia, catalogado como uno de los países megadiversos, la importancia de reducir el uso de productos químicos es muy alta. Sin embargo, el uso indiscriminado de estos productos, se ha convertido en un común denominador entre los agricultores de nuestro país, casi llegando a ser algo tradicional entre ellos. 
Por esta razón, es necesario el estudio de otras alternativas de control de plagas como lo son las biológicas, las cuales no generan un impacto ambiental nocivo.

La Universidad Militar Nueva Granada (UMNG) lleva más de 6 años investigando en el campo del control biológico aplicado a la agricultura colombiana. Se han realizado varios proyectos de investigación que permiten a los investigadores de la UMNG consolidarse como pioneros en el uso de alternativas naturales para el control de plagas en diferentes cultivos de la sabana de Bogotá. Dentro de estos proyectos de investigación está el caso de la avispa parasitoide Encarsia formosa Gahan, icono del control biológico moderno en el mundo (Van Lenteren \& Tommasini 2003). Gracias a los diferentes estudios realizados por investigadores de la Facultad de Ciencias Básicas, se creó una unidad de producción masiva de este parasitoide, con el fin de ofrecerla comercialmente como una alternativa efectiva y económica en el control de la mosca blanca de los invernaderos Trialeurodes vaporariorum, plaga de diferentes cultivos de gran importancia económica en Colombia (Quintero et al. 2001).

El mercado de bio-controladores es todavía una zona de la economía en proceso de desarrollo y expansión (Parra et al. 2002). Actualmente en el país no son muchas las empresas que se dedican a la producción a gran escala de enemigos naturales y la gran mayoría de las empresas emergentes, están direccionadas al campo de los bio-plaguicidas de origen microbiano. Esto se debe a que su producción se realiza con métodos muy parecidos a los empleados en la industria de insecticidas químicos (Instituto Colombiano Agropecuario (ICA) 2010).

El proceso general de la cría de E. formosa se basa en las investigaciones realizadas a nivel de control biológico entre las especies involucradas: planta, plaga y enemigo natural. Debido a esto, el estudio de la producción comercial de esta avispa puede realizarse bajo una perspectiva de un sistema tri-trófico (Mills \& Gutiérrez 1999). Cada una de las especies en el sistema ocupa un nivel trófico en el proceso de producción. En el primer nivel se encuentra el organismo productor, que en este caso, son plantas de frijol Phaseolus Vulgaris L, utilizado para albergar a la plaga y en donde ocurre la interacción plaga - controlador. En el segundo nivel se encuentra el consumidor primario Trialeurodes vaporariorum (Westwood) (Hemiptera: Aleyrodidae), plaga de gran importancia agrícola del cultivo de frijol. El último nivel está ocupado por el consumidor secundario, que es el caso de la avispa parasitoide Encarsia formosa Gahan (Hymenoptera: Aphelinidae), enemigo natural de $T$. vaporariorum y objetivo del proceso de producción.

En este trabajo se determinó realizar un análisis de producción del parasitoide Encarsia formosa en la cría mantenida bajo invernadero del Consultorio de la Facultad de Ciencias Básicas de la Universidad Militar Nueva Granada (UMNG), con el fin de establecer, por medio de diferentes herramientas estadísticas, los niveles óptimos de producción así como también los parámetros biológicos necesarios para el correcto funcionamiento de la cría. 


\section{Materiales y métodos}

Este trabajo se realizó en la estación experimental Hacienda Riogrande de la Universidad Militar Nueva Granada, ubicada en el municipio de Cajicá (4 ${ }^{\circ} 56^{\prime} \mathrm{N}$, $74^{\prime \prime} \mathrm{W}$ ) a una altitud de $2580 \mathrm{msnm}$, con temperatura media anual de $14^{\circ} \mathrm{C}$, humedad relativa de $76 \%$ y precipitación promedio registrada de $1300 \mathrm{~mm}$, bajo condiciones de laboratorio e invernadero, durante los meses de agosto a diciembre del año 2010. Durante este periodo se registraron los datos de producción como el número de avispas producidas diariamente en cada lote y el número de avispas liberadas en cada lote de producción.

Se cultivaron plantas de frijol de la variedad bola roja, con el fin de realizar los diferentes experimentos para establecer las relaciones entre parámetros biológicos como el área foliar, las densidades de la plaga y de la avispa, la preferencia de oviposición en la hoja y la relación entre el número de avispas liberadas y el número de avispas producidas. Las plantas provenían de la unidad de propagación de la cría, teniendo estas las mismas condiciones de producción y la misma edad de manera tal que se evitara la variabilidad causada por estos factores.

Durante todo el proceso de la cría del parasitoide, se registraron los datos de producción haciendo conteos diarios de los individuos de E. formosa originados. El registro de datos se realizó por medio de un formato en donde se relacionaban los lotes producidos semanalmente, con las fechas de siembra, trasplante, infestación, parasitación, porcentaje de infestación, cantidad de adultos del parasitoide liberados y cantidad de adultos producidos semanalmente.

Posteriormente, estos datos fueron analizados con el fin de generar estadísticos de producción, así como también para construir gráficas de producción diaria, semanal y mensual de individuos de E. formosa.

\subsection{Diseño experimental}

\section{Determinación del área foliar}

Se midió el área foliar de cada una de las hojas de las plantas utilizadas con el fin de analizar la variación de este dato entre las unidades muestrales. Una unidad de muestra consistía de una planta de frijol sembrada en matera, con dos hojas primarias desarrolladas. Se estableció un tamaño de muestra de 10 plantas de un lote de cría de 40 plantas en total con el fin de calcular el promedio de área foliar.

\section{Relación del área foliar y la densidad de la plaga}

Cuando un número de individuos adultos de mosca blanca cubren la totalidad del área de la hoja, se dice que se tiene un porcentaje de infestación alto o cercano al $100 \%$. Estos adultos son entre machos y hembras que depositan huevos que completan su desarrollo alimentándose de la hoja. Todas las hojas de las unidades experimentales del ensayo se infestaron con este criterio, liberando dentro de una cámara de infestación individuos adultos de mosca blanca hasta alcanzar la 
cobertura total de la hoja. Se permitió una interacción entre la planta y la plaga por 24 horas.

Las plantas infestadas se trasladaron a una jaula de madera recubierta con velo suizo y sellado con velcro con el fin de evitar que las unidades experimentales sufrieran variaciones en los datos debido a infestaciones realizadas posteriormente por otros individuos de mosca blanca ajenos al experimento. La jaula se mantuvo en las mismas condiciones ambientales y espaciales en las que se mantienen los lotes de producción de la cría. Al cabo de tres semanas se realizó un conteo de individuos inmaduros de la plaga presentes en cada hoja de cada planta. Para realizar este conteo se dividió la hoja en seis partes, las cuales estaban delimitadas por la presencia de las nervaduras principales de la siguiente forma: la nervadura principal divide la hoja en la mitad estableciendo lado izquierdo (1) y lado derecho (2) de la mimas. Las nervaduras secundarias dividen cada lado de la hoja en tres partes: Borde (A), Medio (B), Centro (C). El conteo de ninfas se hizo en cada una de estas partes y se utilizó un ANOVA para determinar si existe alguna preferencia de oviposición por parte de la mosca blanca en la hoja. El diseño consistió en un DCA usando cada parte de la hoja como un tratamiento y cada hoja de la planta como una repetición. En total se contó con 3 tratamientos y 10 repeticiones para un total de 27 grados de libertad de error experimental. También se consideró cada lado de la hoja como un tratamiento y se realizó un ANOVA para establecer alguna diferencia entre los dos lados. Se realizó una regresión lineal entre el área foliar y el número de ninfas contadas en cada hoja con el fin de encontrar una relación entre estos dos factores.

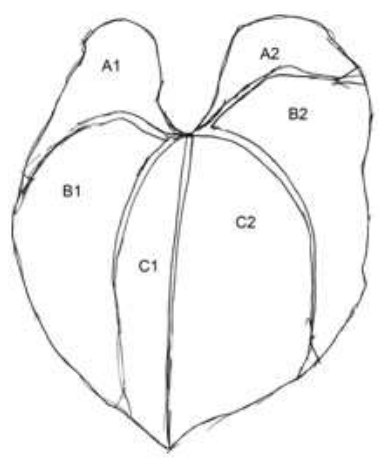

Figura 1: Esquema de una hoja primaria de frijol visto por el envés. $A=$ Borde, $B=$ Medio, $C=$ Centro. $1=$ Izquierda, $2=$ Derecha.

Relación entre el porcentaje de parasitación y el número de individuos de la plaga y el enemigo natural

Se calculó en cada una de las hojas de las plantas del experimento anterior, la cantidad de individuos de E. formosa que se debían liberar para cumplir con una relación de 1:17 (1 adulto del parasitoide por cada 17 individuos inmaduros de 
la plaga). Esta proporción es la recomendada en la literatura y con la cual se obtiene un máximo de parasitación del $85 \%$. Después de establecer el número de individuos de E. formosa según la proporción 1:17, se liberaron las avispas en las hojas de frijol infestadas con mosca blanca. Al cabo de dos semanas se contaron los individuos inmaduros de mosca que habían sido controlados por la avispa.

Utilizando los datos obtenidos en los lotes de producción durante el tiempo en que se desarrolló el trabajo, se calculó la eficiencia de producción como el número de individuos de la avispa generados a partir de individuos usados, es decir, cuántas nuevas avispas se producen por cada parental usada en la cría. Se realizó un análisis de regresión entre la eficiencia de producción y el número de avispas liberadas.

\section{Resultados y discusión}

\subsection{Relación entre el área foliar y el número de individuos inmaduros de la plaga}

La tabla 1 muestra los valores de área foliar de cada una de las hojas de las plantas analizadas, el número de ninfas de tercer instar registrado en cada una de las hojas, el total de individuos por planta y el número de individuos por $\mathrm{cm}^{2}$.

Tabla 1: Datos registrados en el experimento de infestación y promedios calculados de cada parámetro.

\begin{tabular}{|c|c|c|c|c|c|}
\hline Planta & Hoja & $\begin{array}{c}\text { Área Foliar } \\
\mathrm{cm}^{2}\end{array}$ & No. Individuos & $\begin{array}{c}\text { Individuos } \\
\text { por } \mathrm{cm}^{2}\end{array}$ & $\begin{array}{c}\text { Total indvs. } \\
\text { en la planta }\end{array}$ \\
\hline $\mathbf{1}$ & Hoja 1 & 130,25 & 2885 & 22,15 & 6910 \\
& Hoja 2 & 135,10 & 4025 & 29,79 & \\
$\mathbf{2}$ & Hoja 1 & 133,11 & 5246 & 39,41 & 10630 \\
& Hoja 2 & 108,64 & 5384 & 49,55 & \\
$\mathbf{3}$ & Hoja 1 & 99,40 & 1240 & 12,47 & 3928 \\
& Hoja 2 & 148,37 & 2668 & 18,12 & \\
$\mathbf{4}$ & Hoja 1 & 102,40 & 2626 & 25,64 & 5232 \\
& Hoja 2 & 115,46 & 2606 & 22,57 & \\
$\mathbf{5}$ & Hoja 1 & 105,79 & 2578 & 24,37 & 5732 \\
& Hoja 2 & 101,91 & 3154 & 30,95 & \\
\hline $\mathrm{N}=10$ & Media & 118 & 3243 & 27,5 & 6486 \\
\hline
\end{tabular}

La media del área foliar registrada en el experimento fue de $118 \mathrm{~cm}^{2}$. La media del conteo de ninfas de tercer instar de $T$. vaporariorum por hoja fue de 3243 . La media de individuos por área fue de 27,5 ninfas de tercer instar por $\mathrm{cm}^{2}$.

Los datos del área foliar y el número de ninfas de tercer instar se analizaron en un modelo de regresión lineal simple por medio del paquete estadístico SAS v. 9.2 (SAS INSTITUTE INC 2008) utilizando el procedimiento REG especificando un modelo de número de individuos contra área foliar. El valor de $\operatorname{Pr}>\mathrm{F}$ de la 
regresión fue de 0,3961 dando a entender que no hay una relación entre el tamaño del área foliar y le número de individuos.

En la tabla 2 se muestran algunos parámetros estadísticos de las variables de área foliar, la cantidad de individuos por hoja y los individuos por $\mathrm{cm}^{2}$.

Tabla 2: Medidas de dispersión de las variables de Área foliar, Individuos por $\mathrm{cm}^{2}$ y Total Individuos por hoja.

\begin{tabular}{|c|c|c|c|}
\hline & Área foliar $\mathrm{cm}^{2}$ & Indiv.cm & \\
\hline Indiv.hoja & \\
\hline Media & 118,044 & 27,50323533 & 3243,2 \\
Error típico & 5,460792014 & 3,37548109 & 406,778886 \\
Desv. Estándar & 17,26854059 & 10,67420844 & 1286,34778 \\
Varianza & 298,2024942 & 113,9387259 & 1654690,62 \\
Mínimo & 99,399 & 12,4749746 & 1240 \\
Máximo & 148,368 & 49,55498081 & 5384 \\
Suma & 1180,44 & 275,0323533 & 32432 \\
N & 10 & 10 & 10 \\
\hline Coef. Variación & 0,146289016 & 0,388107374 & 0,39662919 \\
\hline
\end{tabular}

Teniendo en cuenta el coeficiente de variación se puede determinar que entre las hojas utilizadas en el experimento, la variabilidad del dato de área foliar fue de alrededor de $15 \%$. Sin embargo, para las mismas hojas, el coeficiente de variación entre los individuos y el total de individuos en una hoja superó ese valor, siendo de $39 \%$ y $40 \%$ respectivamente. A pesar de que la consideración general es que a mayor área foliar habrá un mayor número de individuos, según los resultados encontrados en este trabajo no se puede hacer una relación entre estos dos parámetros.

\subsection{Diferencias de infestación entre zonas de la hoja}

Durante el experimento de infestación se registró el número de individuos inmaduros de la plaga que se encontraban en cada una de las seis secciones de la hoja. Se calculó la media de cada uno de los sectores tomando como tamaño de la muestra las 10 hojas que se midieron del experimento.

En la figura 2 se comparan los promedios del número de individuos en cada zona de la hoja. Como se puede observar, la zona correspondiente al borde de la hoja tiene un promedio de individuos bastante menor en comparación a las zonas más internas. Es posible que la plaga prefiera las zonas internas de la hoja debido a causas alimenticias o de protección.

Se realizaron pruebas de análisis de varianza para cada uno de los siguientes diseños experimentales: primero tomando como tratamientos los lados de la hoja (Izquierdo 1, Derecho 2); segundo tomando como tratamientos cada sección de la hoja (A1, A2, B1, B2...); y por último tomando como tratamientos las zonas simétricas de la hoja borde, medio y centro (A, B y C). 


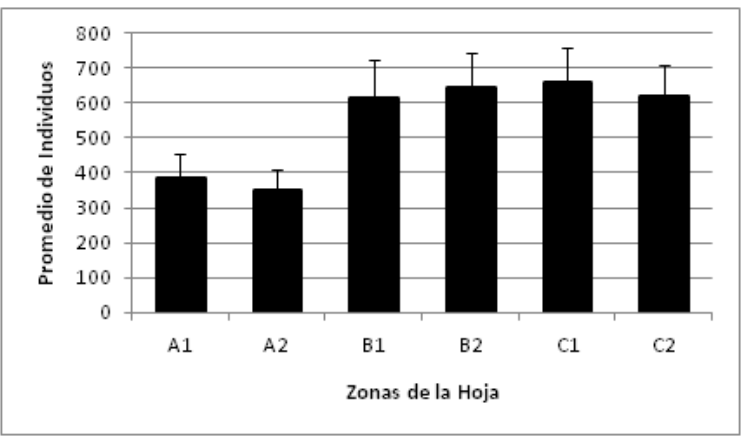

Figura 2: Comparativa del promedio de individuos en cada zona de la hoja.

El análisis de varianza entre el lado izquierdo y derecho de la hoja dio un valor de $\operatorname{Pr}>\mathrm{F}$ de 0,8822 demostrando que no existe una preferencia de oviposición en alguno de estos lados de la hoja (tabla 3 ).

Tabla 3: Análisis de varianza para la oviposición de T. vaporariorum en cada lado de la hoja.Coef. Var. 56,71\%.

\begin{tabular}{cccccc}
\hline Fuente & DF & $\begin{array}{c}\text { Suma de } \\
\text { cuadrados }\end{array}$ & $\begin{array}{c}\text { Cuadrado de } \\
\text { la media }\end{array}$ & F-Valor & Pr $>$ F \\
\hline Modelo & 1 & 4752,600 & 4752,600 & 0,05 & 0,8222 \\
Error & 58 & 5411727,800 & 93305,652 & & \\
Total corregido & 59 & 5416480,400 & & & \\
\hline
\end{tabular}

Como se puede observar en la tabla 4, los promedios de individuos en cada lado de la hoja fueron muy parecidos, dando a entender que cuando se infesta una hoja con una cobertura del $100 \%$ del área, con individuos adultos de mosca blanca, el comportamiento de oviposición es parecido en los dos lados de la hoja.

Tabla 4: Prueba de Agrupamiento de medias de Tukey para Lado 1 Izq. Lado 2 Der.

\begin{tabular}{cccc}
\hline $\begin{array}{c}\text { Tukey } \\
\text { Agrupamiento }\end{array}$ & Media & N & Lado \\
\hline A & 547,50 & 30 & Lado1 \\
A & & & \\
A & 529,70 & 30 & Lado2 \\
\hline
\end{tabular}

Cuando se realizó el análisis de varianza (tabla 5) tomando como tratamiento las zonas Borde (A), Medio (B) y Centro (C) se encontró que existe una diferencia significativa entre las medias de éstas. Según la tabla 6, la prueba de Tukey agrupó las zonas Centro y Medio en un solo grupo dejando la zona Borde en otro grupo aparte. Se puede concluir que existe una tendencia de oviposición de $T$. 
vaporariorum hacia el centro de la hoja. Xu (1985) expone que esta tendencia se debe a la preferencia alimenticia de los adultos por buscar las venaciones, en donde se encuentran altas fuentes de nitrógeno.

Tabla 5: Análisis de varianza de las medias de cada zona de la planta. Borde, Medio y Centro.

\begin{tabular}{cccccc}
\hline Fuente & DF & $\begin{array}{c}\text { Suma de } \\
\text { cuadrados }\end{array}$ & $\begin{array}{c}\text { Cuadrado de } \\
\text { la media }\end{array}$ & F-Valor & Pr $>$ F \\
\hline Modelo 2 & 2 & 1036482,30 & 518241,150 & 6,75 & 0,0023 \\
Error & 57 & 4377370,55 & 76795,975 & & \\
Total corregido & 59 & 5413852,85 & & & \\
\hline
\end{tabular}

Tabla 6: Prueba de Agrupamiento de medias de Tukey para las zonas A=Borde, $B=$ Medio y $C=$ Centro.

\begin{tabular}{cccc}
\hline $\begin{array}{c}\text { Tukey } \\
\text { Agrupamiento }\end{array}$ & Media & N & Lado \\
\hline A & 634,60 & 20 & C \\
A & & & \\
A & 629,35 & 20 & B \\
B & 353,20 & 20 & A \\
\hline
\end{tabular}

En la tabla 7, el análisis de varianza para cada sector de la hoja (A1, A2, B1, B2) muestra que hay diferencia en algunos de estos tratamientos. Sin embargo, el valor de P fue muy cercano a 0,05 por lo cual la prueba de Tukey (no mostrada) agrupó todos los tratamientos en uno solo.

Tabla 7: Análisis de varianza de las medias de cada sector de la planta. Seis sectores en total.

\begin{tabular}{cccccc}
\hline Fuente & DF & $\begin{array}{c}\text { Suma de } \\
\text { cuadrados }\end{array}$ & $\begin{array}{c}\text { Cuadrado de } \\
\text { la media }\end{array}$ & F-Valor & Pr $>$ F \\
\hline Modelo & 5 & 985589,950 & 197117,990 & 2,39 & 0,0496 \\
Error & 54 & 4450955,300 & 82425,098 & & \\
Total corregido & 59 & 5436545,250 & & & \\
\hline
\end{tabular}

\subsection{Relación entre el porcentaje de parasitación y el número de individuos de la plaga y el enemigo natural}

En la tabla 8 se resume la producción de total de la cría en los meses de agosto a diciembre. Como se puede observar, el valor más alto de producción se obtuvo en la semana de cosecha número 43. La relación de parasitoides liberados por el número de individuos producidos para este lote fue de 6,3. La relación máxima de producidos por liberados fue 8,9 nuevos individuos por cada parasitoide liberado. 
Tabla 8: Producción Semanal de individuos (momias + adultos) durante agosto diciembre 2010.

\begin{tabular}{cccccc}
\hline $\begin{array}{c}\text { Número } \\
\text { del lote }\end{array}$ & $\begin{array}{c}\text { Semana } \\
\text { siembra }\end{array}$ & $\begin{array}{c}\text { Semana } \\
\text { cosecha }\end{array}$ & $\begin{array}{c}\text { Avispas } \\
\text { liberadas }\end{array}$ & $\begin{array}{c}\text { Individuos } \\
\text { producidos }\end{array}$ & $\begin{array}{c}\text { Relación } \\
\text { Prod/Libera }\end{array}$ \\
\hline $276-280$ & 24 & 34 & 24,000 & 42,936 & 1,8 \\
$281-285$ & 25 & 35 & 22,600 & 33,062 & 1,5 \\
$286-290$ & 26 & 36 & 26,400 & 41,338 & 1,6 \\
$291-295$ & 27 & 37 & 22,700 & 70,496 & 3,1 \\
$296-300$ & 28 & 38 & 16,000 & 58,829 & 3,7 \\
$301-305$ & 29 & 39 & 10,507 & 66,584 & 6,3 \\
$306-310$ & 30 & 40 & 13,772 & 65,674 & 4,8 \\
$311-315$ & 31 & 41 & 11,759 & 62,299 & 5,3 \\
$316-320$ & 32 & 42 & 23,606 & 68,084 & 2,9 \\
$321-325$ & 33 & 43 & 16,471 & 104,048 & 6,3 \\
$326-330$ & 34 & 44 & 7,156 & 63,638 & 8,9 \\
$331-335$ & 35 & 45 & 12,611 & 92,003 & 7,3 \\
$336-340$ & 36 & 46 & 21,400 & 66,845 & 3,1 \\
$341-345$ & 37 & 47 & 23,131 & 98,619 & 4,3 \\
$346-350$ & 38 & 48 & 26,122 & 62,856 & 2,4 \\
$351-356$ & 39 & 49 & 57,059 & 59,490 & 1,0 \\
$357-361$ & 40 & 50 & 27,730 & 71,800 & 2,6 \\
$362-365$ & 41 & 51 & 29,482 & 59,000 & 2,0 \\
\hline
\end{tabular}

Aragón (2007) reportó que en plantas de tomate cuando se obtenía un porcentaje de control del $77 \%$ de una población de mosca blanca, la relación de individuos controlados por avispas liberadas era de 12,9. Sin embargo, en ese mismo trabajo se encontró un valor máximo de la relación entre ninfas controladas de 23,5 cuando el número de avispas liberadas era el menor, pero a su vez el porcentaje de parasitación también disminuía. Aparentemente esto se debe a un efecto de interferencia entre las avispas. Teniendo en cuenta esto, se realizó un análisis de regresión (figura 3) de la relación entre el número de individuos que se producen por cada avispa liberada, en función de las densidades de la avispa que se usó para producirlas.

En la figura 3 se muestra una tendencia en la cuál el número de individuos que se producen por cada parasitoide liberado (producidos/liberados) disminuye cuando las densidades del parasitoide son más altas. Esto confirma el posible efecto de interferencia que se produce en cada lote cuando se liberan altas poblaciones de la avispa (Hoddle et al. 1998). Se recomienda usar menos avispas para la producción de la cría, lo cual se traduce también en una reducción en los costos de producción y en un aumento de la ventas, como también en una eficiencia en los procesos productivos. El modelo fue generado por el paquete estadístico SAS v. 9.2 (SAS INSTITUTE INC 2008) y sus resultados se observan en la figura 4. 


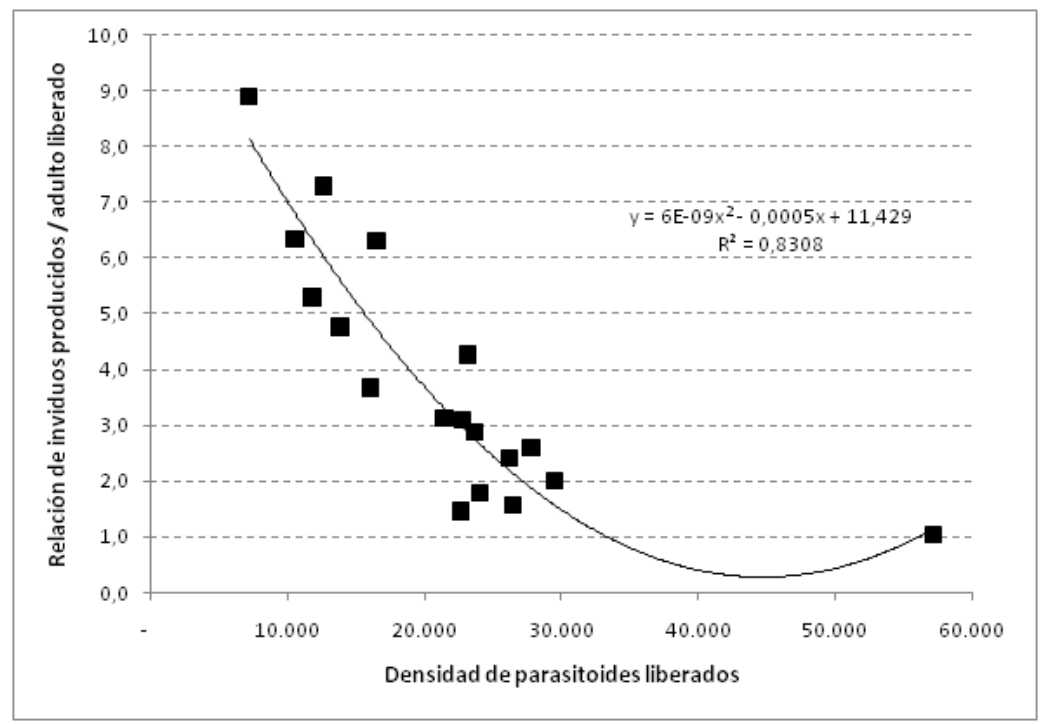

Figura 3: Relación del número de individuos producidos por individuo liberado de E. formosa en función de la densidad de individuos liberados.

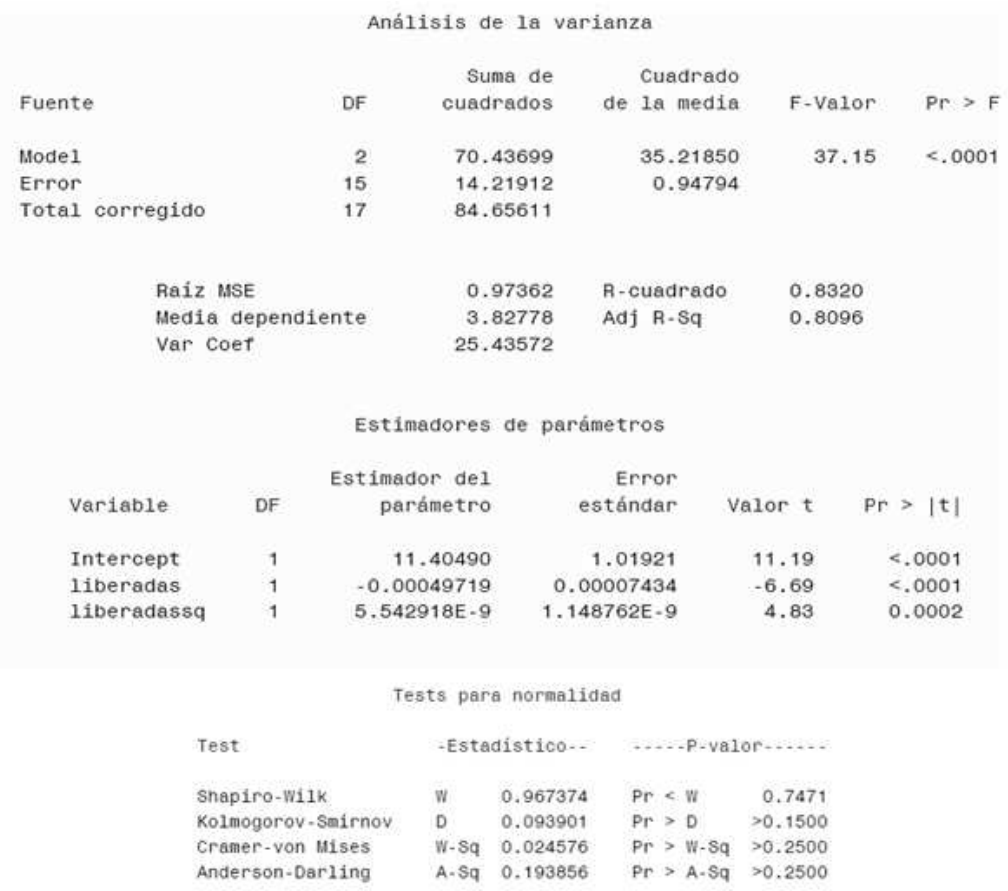

Figura 4: Estadísticas del modelo de regresión ajustado.

Comunicaciones en Estadística, diciembre 2011, Vol. 4, No. 2 


\section{Conclusiones}

El análisis de regresión lineal demostró que no hay una relación entre el área foliar de la hoja y el número de individuos de la plaga que se encuentra en ella, a pesar de que la concepción original es que entre más grande sea una hoja mayor número de individuos tendrá. Esto se debe a que la biología de la plaga no tiene en cuenta el área de la hoja sino la calidad del alimento. Este mismo factor hace que la plaga tenga una preferencia marcada para alimentarse al interior de la hoja, como lo demostró el análisis de varianza. Gracias al análisis de regresión se pudo demostrar estadísticamente la teoría biológica según la cual se establece que existe un efecto de competencia entre individuos de la avispa cuando se liberan grandes cantidades de estas para el control de la plaga. Como se pudo visualizar en este trabajo, el análisis estadístico es una herramienta esencial en los procesos de producción, la cual no sólo permite resolver dudas acerca de la situación biológica de la especies involucradas, sino también encontrar y resolver problemas durante los procesos de producción.

Recibido: 8 de marzo de 2011 Aceptado: 1 de septiembre de 2011

\section{Referencias}

Aragón, S. (2007), Criterios de liberación y evaluación de la efectividad de Encarsia formosa Gahan (Hymenoptera: Aphelinidae) para el control de la mosca blanca Trialeurodes vaporariorum (westwood) (hemiptera: Aleyrodidae) en cultivos comerciales de tomate bajo invernadero, Tesis de maestría, Facultad de Ciencias Básicas. Universidad Militar Nueva Granada.

Hoddle, M. S., van Driesche, R. G. \& Sanderson, J. P. (1998), 'Biology and use of the whitefly parasitoid Encarsia formosa', Annual Review of Entomology 43, 645-669.

Instituto Colombiano Agropecuario (ICA) (2010), Listado de empresas registradas de bioinsumos y extractos vegetales a diciembre de 2010 .

Mills, N. J. \& Gutiérrez, A. P. (1999), Biological control of insect pests: a tritrophic perspective, In: Theoretical Approaches to Biological Control, Hawkins, B. A.; Cornell, H. V (Ed.) Cambridge University Press, Cambridge, UK, pp. 89-102.

Parra, J., Botelho, P. S., Bento, B. \& Bento, B. (2002), 'Controle biológico no brasil: Parasitóides e predadores', Manole 1.

Quintero, C., Rendón, F., García, J., Cardona, C., López-Ávila, A. \& Hernández, P. (2001), 'Especies y biotipos de moscas blancas (Homoptera: Aleyrodidae) en cultivos semestrales de Colombia y Ecuador', Revista Colombiana de Entomología 27, 27-31. 
Análisis estadístico de la producción de un biocontrolador de la mosca blanca 107

SAS INSTITUTE INC (2008), SAS® system for Microsoft $® \quad$ Windows®, v. 9.2 (TSMO).

Van Lenteren, J. \& Tommasini, M. (2003), Mass Production, Storage, Shipment and Release of Natural Enemies. 181-189. in van Lenteren J.C. et al. Quality Control and Production of Biological Control Agents. Theory and Testing Procedurs, CABI International.

Xu, R.-M. (1985), 'Dynamics of within-leaf spatial distribution patterns of greenhouse whiteflies and the biological interpretations', Journal Applied Ecology 22, 63-72. 